\title{
Robust Doubly Protected Estimators for Quantiles with Missing Data.
}

\author{
Julieta Molina $^{(1)}$, Mariela Sued ${ }^{(2)}$, Marina Valdora ${ }^{(1)}$, Víctor Yohai ${ }^{(2)}$ \\ (1) Universidad de Buenos Aires, (2) Universidad de Buenos Aires and Conicet.
}

October 2, 2018

\begin{abstract}
Doubly protected methods are widely used for estimating the population mean of an outcome $Y$ from a sample where the response is missing in some individuals. To compensate for the missing responses, a vector $\mathbf{X}$ of covariates is observed at each individual, and the missing mechanism is assumed to be independent of the response, conditioned on $\mathbf{X}$ (missing at random). In recent years, many authors have turned from the estimation of the mean to that of the median, and more generally, doubly protected estimators of the quantiles have been proposed, under a parametric regression model for the relationship between $\mathbf{X}$ and $Y$ and a parametric form for the propensity score. In this work, we present doubly protected estimators for the quantiles that are also robust, in the sense that they are resistant to the presence of outliers in the sample. We also flexibilize the model for the relationship between $\mathbf{X}$ and $Y$. Thus we present robust doubly protected estimators for the quantiles of the response in the presence of missing observations, postulating a semiparametric regression model for the relationship between the response and the covariates and a parametric model for the propensity score.
\end{abstract}

Keywords: missing data, quantile estimation, doubly protected estimator, robust estimator, semiparametric regression model.

\section{Introduction}

The problem of estimating the mean of a random variable $Y$ from an incomplete data set under the missing at random (MAR) assumption has attracted the attention of the statistical community during the last decades. 
MAR establishes that the variable of interest $Y$ and the response indicator $A$ are conditionally independent given an always observed vector $\mathbf{X}$ of covariates. Most of the existing proposals are based on three different approaches : inverse probability weighted (IPW), outcome regression (OR) and doubly protected (DP) methods.

Inverse probability weighted methods are based on the estimation of the propensity score, denoted by $\pi(\mathbf{X})$, which is defined as the response probability given $\mathbf{X}$. The estimators obtained using this methodology are, essentially, weighted means of observed responses. The weights are determined as the inverse of the estimated propensity scores and their aim is to compensate for the missing observations. Different approaches have been considered for the estimation of the propensity score used in the construction of IPW estimators, for example, logistic regression models [1, 2] and splines [3].

Outcome regression methods require the estimation of the regression function $g(\mathbf{X})=\mathbb{E}(Y \mid \mathbf{X})$. The estimators for $\mathbb{E}(Y)$ built by these techniques average predicted values computed using the estimated regression function. Methods to estimate $g(\mathbf{X})$ include linear regression [4, kernel smoothing [5], semiparametric estimation [7, 8] and local polynomials [9].

IPW procedures are consistent for $\mathbb{E}(Y)$ whenever the propensity score is properly estimated. For instance, in the parametric setting, this requires a correctly specified model for $\pi(\mathbf{X})$. Similarly, OR estimators are consistent provided the predicted values are based on a consistent estimation of the regression function. In a parametric framework, this means that the postulated model for the regression function $g(\mathbf{X})$ should be correct. Doubly protected estimators, also known as doubly robust, combine IPW and OR methods providing consistent estimators for $\mathbb{E}(Y)$ when either the model for the propensity score or the model for the regression function is correct, without having to specify in advance which of them holds. Thus, we get consistent estimators in the union of both the model for the propensity and the model for the regression function. In-depth analysis and examples of doubly protected methods are given in [2],[10], [11], [12], and [13].

Besides the estimation of $\mathbb{E}(Y)$, many authors have recently begun to apply these methods to the estimation of the quantiles of the distribution of $Y$ in the described MAR context. Many of them use the available techniques just described to estimate the distribution function $F_{0}$ of $Y$ by $\widehat{F}_{n}$. Then, the quantiles of $\widehat{F}_{n}$ are used to estimate those of $F_{0}$. Cheng and Chu [14, proposed a Nadaraya-Watson estimator for the conditional distribution of $Y$ given $\mathbf{X}$ and used it to derive a non parametric estimator for the distribution function $F_{0}$. Yang, Kim and Shin [15] presented an imputation method for estimating the quantiles. Imputed data sets are constructed generating plausible values to represent the uncertainty about missing responses. The final 
quantile estimator is obtained combining those computed at each of the multiple imputed data sets. Wang and Qin ([16]) proposed to estimate $F_{0}$ using IPW techniques but estimating the propensity score via kernel regression. Díaz I. 17] proposed to estimate $F_{0}$ under a semiparametric model, using doubly protected techniques by targeted maximum likelihood procedures.

Besides the estimation of the quantiles of $F_{0}$, other location parameters have been considered and some of them also deal with the presence of outliers in the sample, providing robust methods for estimating the parameter of interest. For instance, Bianco et al. [18] used IPW techniques, imputing non parametric estimators of the propensity score $\pi(\mathbf{X})$, and also regression procedures under a semiparametric partially linear regression model to construct location estimators. Asymptotic properties of the estimators involved in these proposals are presented in Bianco et al. [19]. Sued and Yohai [20] also deal with the estimation of the entire distribution of $Y$ considering a semiparametric regression model. Predicted values are combined with observed residuals to emulate a complete data set, based on which one can compute any desired estimator. A robust fit of the regression model is used to take care of anomalous observations. This proposal consistently estimates any parameter defined through a weakly continuous functional at the response's distribution.

Causal inference is an area where missing data inevitably occurs because counterfactual variables may never be observed simultaneously. A large amount of procedures have been designed in such a framework. Among them, we can cite the work by Lunceford and Davidian [10] and the work by Zhang et al. [21] on quantile estimation. Lunceford and Davidian proposed modified IPW estimators in the causal setting, achieving a higher precision compared to the classical ones. Zhang et al. presented several proposals for estimating the distribution function $F_{0}$, all of them based on parametric models for the propensity score or for the conditional distribution of $Y$ given $\mathbf{X}$. They also constructed doubly protected estimators for $F_{0}$ and, therefore, for the quantiles. No protection is provided against the effect of outliers, while the regression framework is entirely parametric.

All the doubly protected proposals to estimate the distribution function $F_{0}$ when $Y$ is missing in some individuals considered up to now are very sensitive to anomalous observations. This is due to the fact that they are based on least squares fits and/or in standard maximum likelihood techniques. In this work, we introduce resistant doubly protected estimators for the distribution function of a scalar outcome that is missing by happenstance on some individuals under a parametric model for the propensity score and a semiparametric regression model for the relation between the outcome and the covariates, assuming missing at random responses. 
The paper is organized as follows. Section 2 introduces the classical procedures to estimate the mean of an outcome $Y$ missing at random. In Section 3 we adapt these procedures to the estimation of $F_{0}$, the distribution function of $Y$ and briefly discuss on the importance of robustness in statistics. In Section 4 we establish the required conditions to get a doubly protected estimator for the percentiles and present a robust doubly protected estimator of the $p$ - quantiles of $F_{0}$. In order to assess the performance of our estimating procedure, in Section 5 we present the results of a Monte Carlo simulation study. In Section 6, a real data set is used to compare the performance of many of the existing techniques. The proofs are presented in the Appendix.

\section{Estimating the mean of $Y$.}

Let us start considering the problem of estimating $\mu=\mathbb{E}(Y)$ based on a sample $\left(X_{1}, A_{1}, Y_{1}\right), \ldots,\left(X_{n}, A_{n}, Y_{n}\right)$, distributed as $(X, A, Y)$, where $Y$ is missing by happenstance on some subjects. To compensate for the missing responses, a vector of covariates $\mathbf{X} \in \mathbb{R}^{p}$ is available for each individual. Moreover, we assume that data are missing at random (MAR) [22]. MAR establishes that the missing mechanism is not related to the response of interest and is only related to the observed vector of covariates. To give a formal definition, let $A$ be the indicator of whether or not $Y$ is missing, i.e., $A=1$ if $Y$ is observed, and $A=0$ when $Y$ is missing. Mathematically, MAR establishes that $\mathbb{P}(A=1 \mid \boldsymbol{X}, Y)=\mathbb{P}(A=1 \mid \boldsymbol{X})=\pi(\mathbf{X}) \cdot \pi(\boldsymbol{X})$ is known in the literature as the propensity score [23].

\subsection{Inverse probability weighted estimators.}

Under the described framework, $\mathbb{E}(Y)$ can be represented in terms of the distribution of the observed data as $\mathbb{E}(Y)=\mathbb{E}\{A Y / \pi(\mathbf{X})\}$. This representation motivates the so-called Horvitz and Thompson [24] estimator of $\mu$, defined by

$$
\widehat{\mu}(\widehat{\pi})=\mathbb{P}_{n}\left\{\frac{A Y}{\widehat{\pi}_{n}(\mathbf{X})}\right\},
$$

where $\widehat{\pi}_{n}(\mathbf{X})$ is a consistent estimator of $\pi(\mathbf{X})$, and $\mathbb{P}_{n}$ is the empirical mean operator, i.e. $\mathbb{P}_{n} V=n^{-1} \sum_{i=1}^{n} V_{i}$. Notice that the observed responses are weighted according to the inverse of the estimated probability of $A=1$ given $\mathbf{X}$, justifying the appellative "inverse probability weighted" (IPW) for such procedures. Moreover, those observed responses corresponding to low values of the estimated propensity score are highly weighted since they should 
compensate for the high missing rate associated to such a level of covariates. For more details see [25].

Different proposals to estimate $\pi(\mathbf{X})$ give rise to diverse estimators of $\mathbb{E}(Y)$, according to (1). Nonparametric estimators of the propensity score have been considered by Little and An [3] and, in a causal context, by Hirano, Imbens and Ridder [26].

In order to assume MAR, the vector $\mathbf{X}$ is typically high dimensional, and therefore, in practice, non parametrical estimation of the propensity score is infeasible because of the so-called curse of dimensionality (see [27]). For this reason the propensity score is often estimated postulating a parametric working model, assuming that

$$
\pi(\mathbf{X})=\pi\left(\mathbf{X} ; \gamma_{0}\right)
$$

where $\boldsymbol{\gamma}_{0} \in \mathbb{R}^{q}$ is an unknown $q$-dimensional parameter and $\pi(\cdot ; \cdot): \mathbb{R}^{p} \times \mathbb{R}^{q} \rightarrow$ $[0,1]$ is a known function. The maximum-likelihood (ML) estimator of $\boldsymbol{\gamma}_{0}$ can be obtained from $\left(\mathbf{X}_{1}, A_{1}\right), \ldots,\left(\mathbf{X}_{n}, A_{n}\right)$, and will be denoted by $\widehat{\gamma}_{n}$. Then, we can estimate $\pi(\mathbf{X})$ by

$$
\widehat{\pi}_{n}(\mathbf{X}):=\pi\left(\mathbf{X} ; \widehat{\gamma}_{n}\right)
$$

Generalized linear models (GLM) are very popular in this setting. These models postulate that $\pi\left(\mathbf{X} ; \boldsymbol{\gamma}_{0}\right)=\phi\left(\boldsymbol{\gamma}_{0}^{t} \mathbf{X}\right)$, where $\phi$ is a strictly increasing cumulative distribution function. In particular, the linear logistic regression model for the propensity score is obtained by choosing $\phi(u)=\exp (u) /\{1+$ $\exp (u)\}$ (see [2] ).

\subsection{Outcome regression estimators.}

To construct IPW estimators, we model the relation between the missing mechanism and the covariates and we do not make assumptions on the relation between the outcome and the covariates. To construct regression estimators for $\mathbb{E}(Y)$, we estimate the regression function of $Y$ on $\mathbf{X}$ and average predicted values. More precisely, under the missing at random assumption we have that $Y|\mathbf{X} \sim Y|(\mathbf{X} ; A=1)$ and therefore the regression function $g(\mathbf{X})=\mathbb{E}(Y \mid \mathbf{X})$ satisfies $g(\mathbf{X})=\mathbb{E}(Y \mid \mathbf{X} ; A=1)$. In this way we arrive at a second representation for $\mathbb{E}(Y)$ based on the observed data through the regression function: $\mathbb{E}(Y)=\mathbb{E}\{g(\mathbf{X})\}$. This alternative characterization for $\mathbb{E}(Y)$ invites us to estimate it by averaging predicted values:

$$
\widehat{\mu}\left(\widehat{g}_{n}\right)=\mathbb{P}_{n}\left\{\widehat{g}_{n}(\mathbf{X})\right\},
$$


where $\widehat{g}_{n}(\mathbf{X})$ is any consistent estimator of $g(\mathbf{X})$. Different ways to estimate $g(\mathbf{X})$ result in different estimators according to (4). A non parametric proposal for $\widehat{g}_{n}(X)$ is given in Cheng [5], using kernel regression estimation duly adapted to the missing data context, in order to estimate the regression function. Imbens Newey and Ridder [6] proposed non parametric estimation for both the propensity score and the regression function.

In practice, working parametric models are postulated for the regression function to overcome the curse of dimensionality, which is a serious obstacle for non parametric methods (see [27]). A parametric model for the regression function assumes that

$$
g(\mathbf{X})=g\left(\mathbf{X} ; \boldsymbol{\beta}_{0}\right),
$$

where $g(\cdot ; \cdot)$ is a known function and $\boldsymbol{\beta}_{0} \in \mathbb{R}^{r}$ is unknown. The unknown parameter $\boldsymbol{\beta}_{0}$ of the working regression model (5) can be estimated by $\widehat{\boldsymbol{\beta}}_{n}$, using the units with observed responses $Y$ by, for instance, the least squares method. Then, $g(\mathbf{X})$ is estimated with

$$
\widehat{g}_{n}(\mathbf{X}):=g\left(\mathbf{X} ; \widehat{\boldsymbol{\beta}}_{n}\right)
$$

and this expression is imputed in (4) to obtain parametric regression estimators of $\mathbb{E}(Y)$.

Kang and Shafer [2] review the regression estimator of $\mu$ that results from considering a linear model for the regression function: $g(\mathbf{X} ; \boldsymbol{\beta})=\boldsymbol{\beta}^{t} \mathbf{X}$. A comprehensive overview of parametric regression estimators is given in [1].

We also want to mention that another way to deal with the curse of dimensionality is to consider intermediate structures like additive models or semiparametric models for the regression function. See, for example, [7] and [28].

\subsection{Doubly protected estimators.}

Estimators based on inverse probability weighting, as presented in (1) are consistent for $\mu$ as far as the propensity score $\pi(\mathbf{X})$ is consistently estimated. This approach leads to a well specified model for $\pi(\mathbf{X})$ in the parametric case. On the other hand, regression estimators, as presented in (4), are consistent when the regression function is properly estimated and thus, the regression model is assumed to be correctly specified.

To sum up, each procedure forces us to choose in advance what to model in order to decide which estimator should be used to consistently estimate $\mathbb{E}(Y)$. Typically no one knows which model is more suitable, generating a debate on which approach should be used. To end this controversy, estimators which are consistent for $\mathbb{E}(Y)$ whenever, at least, one of the two models 
is correct were proposed. Such estimators confer more protection to model misspecification than IPW or OR estimators, which are consistent only when the corresponding assumed model holds. Since these estimators are consistent for $\mathbb{E}(Y)$ as long as one of the models succeeds, they are called doubly protected estimators.

Doubly protected estimators were discovered by Robins et al. [30, 8], while studying augmented IPW estimators (AIPW). Some years later, Scharfstein et al. [31 showed that some AIPW estimators have the double protection property. To motivate these estimators, they obtained the following expression for the mean that holds assuming MAR and that either $p(\mathbf{X})=$ $\mathbb{P}(A=1 \mid \mathbf{X})$ or $r(\mathbf{X})=\mathbb{E}(Y \mid \mathbf{X})$ hold:

$$
\mu=\mathbb{E}\left\{\frac{A Y}{p(\mathbf{X})}\right\}-\mathbb{E}\left[\left\{\frac{A}{p(\mathbf{X})}-1\right\} r(\mathbf{X})\right] .
$$

Therefore, doubly protected estimators can be obtained by postulating parametric models $\pi\left(\mathbf{X} ; \boldsymbol{\gamma}_{0}\right)=\mathbb{P}(A=1 \mid \mathbf{X})$ and $g\left(\mathbf{X} ; \boldsymbol{\beta}_{0}\right)=\mathbb{E}(Y \mid \mathbf{X})$, like in (2) and (5), respectively, and estimating $\mu$ by

$$
\widehat{\mu}_{D P}=\mathbb{P}_{n}\left\{\frac{A Y}{\widehat{\pi}_{n}(\mathbf{X})}\right\}-\mathbb{P}_{n}\left[\left\{\frac{A}{\widehat{\pi}_{n}(\mathbf{X})}-1\right\} \widehat{g}_{n}(\mathbf{X})\right]
$$

where $\widehat{\pi}_{n}$ and $\widehat{g}_{n}$ are defined in (3) and (6).

The estimator defined in (8) is doubly protected, and also achieves full efficiency in the AIPW class if the model for the propensity and the model for the regression function are both well specified (see [30] and [8]). An in-depth analysis and examples of doubly-protected methods are given in [10, [11, 12 and [2. For the complete and detailed mathematical theory underlying double protection methodology see [32] and [13].

\section{Estimating the quantiles of the distribution of $Y$}

In this section we move from the estimation of $\mathbb{E}(Y)$ to the estimation of the median of the distribution function of $Y$. More generally, we will focus on the estimation of any quantile of $F_{0}$, the distribution function of $Y$. The $p$-quantile of a distribution $F$ is defined as

$$
T_{p}(F)=\inf \{x: F(x) \geq p\} .
$$

When $p=0.5, T_{0.5}(F)$ is the median of $F$. The representation of the p-quantile given in (9) suggests that it can be estimated by $T_{p}\left(\widehat{F}_{n}\right)$, provided 
$\widehat{F}_{n}$ approximates $F_{0}$. So, to estimate a $p$-quantile of a distribution of $Y$ it is enough to estimate $F_{0}$. Note that $F_{0}(y)=\mathbb{P}(Y \leq y)=\mathbb{E}\left(\mathrm{I}_{\{Y \leq y\}}\right)$. In the next sections, we will provide different proposals of estimation for the distribution function of $Y$, mimicking each of the estimators considered in the previous sections, but to estimate now $\mathbb{E}\left\{\ell_{y}(Y)\right\}$, where $\ell_{y}(Y)=\mathrm{I}_{\{Y \leq y\}}$, in lieu of $\mu=\mathbb{E}(Y)$.

\subsection{IPW estimators of $F_{0}$.}

Under the MAR assumption

$$
F_{0}(y)=\mathbb{E}\left\{\mathrm{I}_{\{Y \leq y\}}\right\}=\mathbb{E}\left\{\frac{A \mathrm{I}_{\{Y \leq y\}}}{\pi(\mathbf{X})}\right\}
$$

and so, if we apply the procedure developed in Section 2.1, we arrive at the following estimator for $F_{0}(y)$

$$
\widehat{F}_{\mathrm{IPW}}(y)=\mathbb{P}_{n}\left\{\frac{A \mathrm{I}_{\{Y \leq y\}}}{\widehat{\pi}_{n}(\mathbf{X})}\right\}
$$

where $\widehat{\pi}_{n}(\mathbf{X})$ is a consistent estimator of the propensity score. A slightly modified version of this estimator, with weights adding up to one, has already been introduced by Bianco et al. [18] and used for estimating any M-location functional of the distribution of $Y . \widehat{F}_{\text {ipw }}$ was also proposed by Zhang et al. [21] for estimating quantiles under a parametric model for the propensity score.

\subsection{Regression Estimators of $F_{0}$}

Regression estimators are constructed based on the following representation

$$
F_{0}(y)=\mathbb{E}\{\mathbb{P}(Y \leq y \mid \mathbf{X})\} .
$$

Thus, we can estimate $F_{0}$ by

$$
\widehat{F}_{\mathrm{REG}}(y)=\mathbb{P}_{n} \widehat{\mathbb{P}}(Y \leq y \mid \mathbf{X})=\frac{1}{n} \sum_{i=1}^{n} \widehat{\mathbb{P}}\left(Y \leq y \mid \mathbf{X}_{i}\right) .
$$

Suppose, for instance, that a generalized linear model is postulated, assuming that $Y \mid \mathbf{X} \sim G_{\boldsymbol{\beta}_{0}{ }^{t} \mathbf{X}}$, where $\left\{G_{\boldsymbol{\theta}}: \boldsymbol{\theta} \in \Theta\right\}$ is an exponential family of univariate distributions, and $\boldsymbol{\theta}$ is the vector of natural parameters. In this case, because of the MAR assumption, $Y \mid(\mathbf{X} ; A=1) \sim G_{\boldsymbol{\beta}_{0}^{t} \mathbf{X}}$ and so $\boldsymbol{\beta}_{0}$ can 
be consistently estimated with $\widehat{\boldsymbol{\beta}}_{n}$, the maximum likelihood estimator under the model using the pairs $\left(\mathbf{X}_{i}, Y_{i}\right)$ with $A_{i}=1$. Then, according to $(13)$, in this particular case, we estimate $F_{0}$ with

$$
\widehat{F}_{\mathrm{GLM}}(y)=\frac{1}{n} \sum_{i=1}^{n} G_{\widehat{\boldsymbol{\beta}}_{n}^{t} \mathbf{x}_{i}}(y) .
$$

Assume now that $Y$ follows a regression model of the form

$$
Y=g(\mathbf{X})+u
$$

where $g$ is the unknown regression function that maps $\mathbb{R}^{p}$ into $\mathbb{R}$, and $u$ is independent of $\mathbf{X}$. Moreover, to guarantee the MAR assumption we require $(\mathbf{X}, A)$ to be independent of $u$. Let $\widehat{g}_{n}(\mathbf{X})$ be a consistent estimator of the regression function, constructed using the pairs $\left(\mathbf{X}_{i}, Y_{i}\right)$ with observed responses $\left(A_{i}=1\right)$. Under the regression model $(15)$, the assumed independence between $u$ and $\mathbf{X}$ implies that $\mathbb{P}(Y \leq y \mid \mathbf{X})=\mathbb{P}\{u \leq y-g(\mathbf{X})\}$ and so, it can be estimated with the empirical distribution of the residuals $\widehat{u}_{j}=Y_{j}-\widehat{g}_{n}\left(\mathbf{X}_{j}\right)$, for $A_{j}=1$, at $y-\widehat{g}_{n}(\mathbf{X})$ :

$$
\widehat{\mathbb{P}}(Y \leq y \mid \mathbf{X})=\widehat{F}_{\text {error }}\left(y-\widehat{g}_{n}(\mathbf{X})\right)=\frac{1}{m} \sum_{j=1}^{n} A_{j} \mathrm{I}_{\left\{\widehat{u}_{j} \leq y-\widehat{g}_{n}(\mathbf{X})\right\}},
$$

where $m$ denotes the number of observed responses, that is $m=\sum_{j=1}^{n} A_{j}$. Combining (16) with (13) we obtain the following estimator for $F_{0}$

$$
\widehat{F}(y)=\frac{1}{n} \sum_{i=1}^{n} \widehat{F}_{\text {error }}\left(y-\widehat{g}_{n}\left(\mathbf{X}_{i}\right)\right)=\frac{1}{n m} \sum_{i, j=1}^{n} A_{j} \mathrm{I}_{\left\{\widehat{u}_{j} \leq y-\widehat{g}_{n}\left(\mathbf{X}_{i}\right)\right\}} .
$$

Note that $\widehat{F}$ assigns mass $1 /(n m)$ to the values

$$
\widehat{Y}_{i j}=\widehat{g}_{n}\left(\mathbf{X}_{i}\right)+\widehat{u}_{j}, \quad 1 \leq i, j \leq n, j ; A_{j}=1,
$$

where predicted values $\widehat{g}_{n}\left(\mathbf{X}_{i}\right)$ are combined with residuals $\widehat{u}_{j}$ to emulate a pseudo-sample of responses $\widehat{Y}_{i j}$, as suggested by equation 15 .

Sued and Yohai [20], proposed a semiparametric regression model for (15), where the regression function is assumed to be in a parametric family: $g(\mathbf{X})=g\left(\mathbf{X} ; \boldsymbol{\beta}_{0}\right)$, with $\boldsymbol{\beta}_{0} \in B \subset \mathbb{R}^{q}$, and $g: \mathbb{R}^{p} \times B \rightarrow \mathbb{R}$ is a known function. No other than a centrality condition, namely symmetry around zero, is imposed on the error term $u$. In fact, Sued and Yohai [20] showed that the centrality condition can be avoided, redefining properly the intercept in the regression model. But, to keep this presentation more accessible, we 
can focus on the centered error case. This gives rise to the estimator $\widetilde{F}$, defined by

$$
\widetilde{F}(y)=\frac{1}{n m} \sum_{i, j=1}^{n} A_{i} \mathrm{I}_{\left\{\widehat{Y}_{i j} \leq y\right\}},
$$

where $\widehat{Y}_{i j}$ are defined as in 17 with $\widehat{g}_{n}(\mathbf{X})=g\left(\mathbf{X} ; \widehat{\boldsymbol{\beta}}_{n}\right)$. The authors proved that $\widetilde{F}$ converges to $F_{0}$, as far as $\widehat{\boldsymbol{\beta}}_{n}$ converges to $\beta_{0}$. In particular, this procedure allows the estimation of the $p$-quantiles of $F_{0}$ with $T_{p}(\widetilde{F})$, where $T_{p}$ is defined in (9).

Model 15) with a linear regression function $g(\mathbf{X} ; \boldsymbol{\beta})=\boldsymbol{\beta}^{t} \mathbf{X}$ and Gaussian errors $\left(u \sim \mathcal{N}\left(0, \sigma^{2}\right)\right)$ fits the GLM framework described at the beginning of this section. In particular, according to (14), we arrive at the following estimator of $F_{0}$

$$
\widehat{F}_{\mathrm{G}}(y)=\frac{1}{n} \sum_{i=1}^{n} \Phi\left(\frac{y-\widehat{\boldsymbol{\beta}}_{n}^{t} \mathbf{X}_{i}}{\widehat{\sigma}}\right),
$$

where $\Phi$ denotes the cumulative distribution of a standard normal random variable. This estimator was studied by Zhang et al. in [21]. We remark the semiparametric nature of the $\widehat{F}_{\mathrm{SY}}$ presented in $(23)$, where no model is assumed for the distribution of the error term $u$.

\subsection{Doubly protected estimators of $F_{0}$}

Replacing $Y$ with $\mathrm{I}_{\{Y \leq y\}}$ in (7), we obtain

$$
F_{0}(y)=\mathbb{E}\left\{\mathrm{I}_{\{Y \leq y\}}\right\}=\mathbb{E}\left\{\frac{A \mathrm{I}_{\{Y \leq y\}}}{p(\mathbf{X})}\right\}-\mathbb{E}\left[\left\{\frac{A}{p(\mathbf{X})}-1\right\} r_{y}(\mathbf{X})\right],
$$

under MAR, and supposing either $p(\mathbf{X})=\mathbb{P}(A=1 \mid \mathbf{X})$ or $r_{y}(\mathbf{X})=\mathbb{P}(Y \leq$ $y \mid \mathbf{X})$ holds. Let $\pi(\mathbf{X})=\mathbb{P}(A=1 \mid \mathbf{X})$ and $g_{y}(\mathbf{X})=\mathbb{P}(Y \leq y \mid \mathbf{X})$. $F_{0}$ can be doubly protectedly estimated through a plug - in procedure inspired in expression (19), by

$$
\widehat{F}(y)=\frac{1}{n} \sum_{i=1}^{n} \frac{A_{i} \mathrm{I}_{\left\{Y_{i} \leq y\right\}}}{\widehat{\pi}_{n}\left(\mathbf{X}_{i}\right)}-\frac{1}{n} \sum_{i=1}^{n}\left\{\frac{A_{i}}{\widehat{\pi}_{n}\left(\mathbf{X}_{i}\right)}-1\right\} \widehat{g}_{y}\left(\mathbf{X}_{i}\right) .
$$

At this point, we turn to a parametric framework to model the propensity score with $\pi(\mathbf{X})=\pi\left(\mathbf{X} ; \boldsymbol{\gamma}_{0}\right)$. We also assume that model 15$)$ holds and that the regression function $g(\mathbf{X})$ satisfies $g(\mathbf{X})=g\left(\mathbf{X} ; \boldsymbol{\beta}_{0}\right)$. Thus, we can deal with a semiparametic regression model, instead of a parametric one, where typically also the distribution of the error term is assumed to belong to a 
parametric family. In this way, using $\widehat{F}_{\text {error }}$ defined in $(16)$, we arrive at the following semiparametric doubly protected estimator for $F_{0}$

$$
\widehat{F}_{\mathrm{DP}-\mathrm{S}}(y)=\frac{1}{n} \sum_{i=1}^{n} \frac{A_{i} \mathrm{I}_{\left\{Y_{i} \leq y\right\}}}{\pi\left(\mathbf{X}_{i} ; \widehat{\boldsymbol{\gamma}}_{n}\right)}-\frac{1}{m n} \sum_{i, j=1}^{n}\left\{\frac{A_{i}}{\pi\left(\mathbf{X}_{i} ; \widehat{\boldsymbol{\gamma}}_{n}\right)}-1\right\} A_{j} \mathrm{I}_{\left\{g\left(\mathbf{X}_{i} ; \widehat{\boldsymbol{\beta}}_{n}\right)+\widehat{u}_{j} \leq y\right\}},
$$

where $\widehat{\boldsymbol{\gamma}}_{n}$ and $\widehat{\boldsymbol{\beta}}_{n}$ are estimators of $\boldsymbol{\gamma}_{0}$ and $\boldsymbol{\beta}_{0}$, respectively.

The GLM presented in Section 3.2 can also be used to impute an estimator of $g_{y}(\mathbf{X})=\mathbb{P}(Y \leq y \mid \mathbf{X})$ in the formula given in (20). For instance, the linear model with Gaussian errors $\left(u \sim \mathcal{N}\left(0, \sigma^{2}\right)\right)$ is a particular case that was already considered in [21], giving rise to the following formula for estimating $F_{0}$ :

$$
\widehat{F}_{\mathrm{DP}-\mathrm{G}}(y)=\frac{1}{n} \sum_{i=1}^{n} \frac{A_{i} \mathrm{I}_{\left\{Y_{i} \leq y\right\}}}{\pi\left(\mathbf{X}_{i} ; \widehat{\gamma}_{n}\right)}-\frac{1}{n} \sum_{i=1}^{n}\left\{\frac{A_{i}}{\pi\left(\mathbf{X}_{i} ; \widehat{\boldsymbol{\gamma}}_{n}\right)}-1\right\} \Phi\left(\frac{y-\widehat{\boldsymbol{\beta}}_{n}^{t} \mathbf{X}_{i}}{\widehat{\sigma}}\right),
$$

where $\Phi$ denotes the cumulative distribution of a standard normal random variable, $\widehat{\beta}_{n}$ is the least square estimator of the regression coefficients, and $\widehat{\sigma}$ estimates the standard deviation of the errors.

\subsection{Robustness}

Atypical observations, called outliers, are common in many real datasets. Classical procedures do not contemplate their existence and therefore their application may lead to wrong conclusions. For instance, the sample mean or the least-squares fit of a regression model, can be very adversely influenced by outliers, even by a single one. Robust methods arise to cope with these atypical observations, mitigating their impact in the final analysis. The median is, probably, the most popular example of a robust procedure to summarize a univariate dataset. More generally, M-location estimators have been developed for such a purpose: a robust location summary of a univariate data set. Thus, moving from the estimation of $\mathbb{E}(Y)$ to that of the median of $F_{0}$ represents a first step in the path towards robustification. However, the methods presented in the previous section, also require regression fits, both to estimate the propensity score and the regression function. In this work we propose to consider a robust alternative for the regression fit of the postulated model that relates $\mathbf{X}$ and $Y$. In fact, when model (5) is combined with a parametric regression function assuming that $g(\mathbf{X})=g\left(\mathbf{X} ; \boldsymbol{\beta}_{0}\right)$, the least-squares fit will be replaced by a robust one. One way to achieve this robustification is to replace the square loss function by a so called $\rho$-function evaluated at the norm 
of standardized residuals. A $\rho$-function, $\rho: \mathbb{R} \rightarrow[0, \infty]$, is assumed to be (i) continuous, (ii) even, (iii) non-decreasing function of $|t|$ and (iv) $\rho(0)=0$. Moreover, in order to deal with high leverage outliers the $\rho$-function should be bounded (see Section 5.4.1 in [41]).

This is the case of M-estimators, both for location and regression problems. In particular, MM-estimators, introduced by Yohai [33 for the linear model, and extended to the non linear case in Fasano et al. 34, combines the highest possible tolerance to the presence of outliers, measured by the breakdown point, with an arbitrarily high efficiency in the case of Gaussian errors.

The use of a robust fit for the regression model to estimate $F_{0}$ has already been considered in 20 . Indeed, they presented $\widehat{F}_{S Y}$, a semiparametric regression estimators as those discussed in Section 3.2, where the regression parameter $\boldsymbol{\beta}_{0}$ is estimated with $\widehat{\boldsymbol{\beta}}_{n}^{\mathrm{R}}$, an MM- estimator. Thus,

$$
\widehat{F}_{S Y}(y)=\frac{1}{n m} \sum_{i, j=1}^{n} A_{i} \mathrm{I}_{\left\{g\left(\mathbf{X}_{j} ; \widehat{\boldsymbol{\beta}}_{n}^{\mathrm{R}}\right)+Y_{i}-g\left(\mathbf{X}_{i} ; \widehat{\boldsymbol{\beta}}_{n}^{\mathrm{R}}\right) \leq y\right\}} .
$$

The authors showed that $T\left(\widehat{F}_{S Y}\right)$ gives rise to a robust method for estimating $T\left(F_{0}\right)$, for any weak-continuous functional $T$ at $F_{0}$.

Robust doubly protected estimations of $F_{0}$ can be obtained by replacing the least squares estimator of $\boldsymbol{\beta}_{0}$ by $\widehat{\boldsymbol{\beta}}_{n}^{\mathrm{R}}$, an MM-estimator. For instance, a robust version of the doubly protected estimator discussed in [21], presented in $(22)$, can be defined as

$$
\widehat{F}_{\text {DP-G-ROB }}(y)=\frac{1}{n} \sum_{i=1}^{n} \frac{A_{i} \mathrm{I}_{\left\{Y_{i} \leq y\right\}}}{\pi\left(\mathbf{X}_{i} ; \widehat{\boldsymbol{\gamma}}_{n}\right)}-\frac{1}{n} \sum_{i=1}^{n}\left\{\frac{A_{i}}{\pi\left(\mathbf{X}_{i} ; \widehat{\boldsymbol{\gamma}}_{n}\right)}-1\right\} \Phi\left(\frac{y-\mathbf{X}_{i}^{t} \widehat{\boldsymbol{\beta}}_{n}^{\mathrm{R}}}{\widehat{s}}\right),
$$

where $\widehat{s}$ is a robust scale of the residuals.

An MM-estimator can also be used to robustify the semiparametric doubly protected estimator $\widehat{F}_{\mathrm{DP}-\mathrm{S}}$, defined in (21). We postpone this construction until next section, where $\widehat{F}_{\mathrm{DP}-\mathrm{S}}$ is slightly modified in order get a consistent procedure. In this way, we will be able to present both a classical and a robust doubly protected estimator of $F_{0}$.

The next step is, naturally, to robustify the estimation of $\boldsymbol{\gamma}_{0}$, the parameter involved in the propensity score. However, this simple approach would not robustify the final estimator. On the contrary, in the presence of outliers in the covariates, extreme values of $\widehat{\pi}_{n}$ are more likely to appear if $\gamma_{0}$ is estimated robustly than otherwise. Resistance to outliers in $A$ seems a difficult problem, whose solution we are still working on and might be the subject of further work. 


\section{Consistency}

The estimator $\widehat{F}_{\mathrm{DP}-\mathrm{S}}(y)$ defined in equation 21 is not a cumulative distribution function of a probability measure. However, it can be associated to a discrete signed measure on $\mathbb{R}$. Moreover, we can decompose it as

$\widehat{F}_{\mathrm{DP}-\mathrm{S}}(y)=\frac{1}{n} \sum_{i=1}^{n} \frac{A_{i} \mathrm{I}_{\left\{Y_{i} \leq y\right\}}}{\widehat{\pi}_{n}\left(\mathbf{X}_{i}\right)}-\frac{1}{n m} \sum_{i, j=1}^{n} \frac{A_{i}}{\widehat{\pi}_{n}\left(\mathbf{X}_{i}\right)} A_{j} \mathrm{I}_{\left\{\widehat{g}_{n}\left(\mathbf{X}_{i}\right)+\widehat{u}_{j} \leq y\right\}}+\frac{1}{n m} \sum_{i, j=1}^{n} A_{j} \mathrm{I}_{\left\{\widehat{g}_{n}\left(\mathbf{X}_{i}\right)+\widehat{u}_{j} \leq y\right\}}$,

with $\widehat{\pi}_{n}(\mathbf{X})=\pi\left(\mathbf{X} ; \widehat{\boldsymbol{\gamma}}_{n}\right)$ and $\widehat{g}_{n}(\mathbf{X})=g\left(\mathbf{X} ; \widehat{\boldsymbol{\beta}}_{n}\right)$. Let $\widehat{F}_{1}(y), \widehat{F}_{2}(y)$ and $\widehat{F}_{3}(y)$ denote the three terms in expression (25). Only the last one of them, $\widehat{F}_{3}$, corresponds to a cumulative distribution function of a probability measure. The total mass of neither the first term, $\widehat{F}_{1}$, nor the second one, $\widehat{F}_{2}$, is equal to one. This issue can be easily corrected normalizing them properly. Let $\widetilde{F}_{1}$ be the normalized correction of $\widehat{F}_{1}$, namely

$$
\widetilde{F}_{1}:=\frac{1}{C_{n}} \sum_{i=1}^{n} \frac{A_{i} \delta_{Y_{i}}}{\widehat{\pi}_{n}\left(\mathbf{X}_{i}\right)} \text {, where } C_{n}:=\sum_{i=1}^{n} \frac{A_{i}}{\widehat{\pi}_{n}\left(\mathbf{X}_{i}\right)},
$$

and $\delta_{s}$ denotes the distribution function of the point mass probability measure concentrated at $s$. To normalize the second term involved in expansion (25), consider

$$
\widetilde{F}_{2 a}:=\frac{1}{C_{n}} \sum_{i=1}^{n} \frac{A_{i} \delta_{\widehat{g}_{n}\left(\mathbf{X}_{i}\right)}}{\widehat{\pi}_{n}\left(\mathbf{X}_{i}\right)}, \quad \widetilde{G}:=\frac{1}{m} \sum_{j=1}^{n} A_{j} \delta_{\widetilde{u}_{j}},
$$

and, therefore, the normalized version of $\widehat{F}_{2}$, is given by

$$
\widetilde{F}_{2}:=\widetilde{F}_{2 a} * \widetilde{G}
$$

where $*$ stands for the convolution operator between two distribution functions. Finally, recalling that $\widehat{F}_{3}$ is already a cumulative distribution function, note that it can be written as

$$
\widehat{F}_{3}=\widehat{F}_{3 a} * \widetilde{G}, \quad \text { with } \quad \widetilde{F}_{3 a}=\frac{1}{n} \sum_{i=1}^{n} \delta_{\widehat{g}_{n}}\left(\mathbf{X}_{i}\right) .
$$

The normalized version of the doubly protected estimator presented in (25) is given by

$$
\widehat{F}_{\mathrm{DP}-\mathrm{NOR}}=\widetilde{F}_{1}-\widetilde{F}_{2 a} * \widetilde{G}+\widetilde{F}_{3 a} * \widetilde{G} .
$$

At this point we want to emphasize that, even though each term of the sum in 30 is a cumulative distribution function, the non convexity of the 
linear combination that defines $\widehat{F}_{\mathrm{DP}-\mathrm{NOR}}$ causes it to fall out of the space of cumulative distribution functions. However, Theorem 2 states that, under assumptions A1-A3 given below, $\widehat{F}_{\text {DP-NOR }}$ is a doubly protected estimator of $F_{0}$ since it converges uniformly to $F_{0}$ almost surely (a.s.), if either $\widehat{\pi}_{n}(\mathbf{X})$ converges to $\pi(\mathbf{X})$ or $\widehat{g}_{n}(\mathbf{X})$ converges to $g(\mathbf{X})$. Even though $\widehat{F}_{\mathrm{DP}-\mathrm{NOR}}$ is not a cumulative distribution function, Lemma 4 says that $T_{p}\left(\widehat{F}_{\mathrm{DP}-\mathrm{NOR}}\right)$ is well defined, with $T_{p}$ as in (9). Finally, the aforementioned theorem states that $T_{p}\left(\widehat{F}_{\text {DP-NOR }}\right)$ converges to $T_{p}\left(F_{0}\right)$ a.s. if either $\widehat{\pi}_{n}(\mathbf{X})$ converges to $\pi(\mathbf{X})$ or $\widehat{g}_{n}(\mathbf{X})$ converges to $g(\mathbf{X})$. Therefore, $T_{p}\left(\widehat{F}_{\mathrm{DP}-\mathrm{NOR}}\right)$ results a doubly robust estimator of the $p$-quantile of the distribution of $Y$, as established in Theorem 2 .

In order to study the asymptotic behaviour of $\widehat{F}_{\mathrm{DP}-\mathrm{NOR}}$, defined in 30, we consider the following assumptions.

- A1: There exists a sequence of random functions $\left(\widehat{\pi}_{n}\right)_{n \geq 1}, \widehat{\pi}_{n}: \mathbb{R}^{p} \rightarrow$ $(0,1)$, depending on $\left(X_{1}, A_{1}\right), \ldots,\left(X_{n}, A_{n}\right)$, i.i.d., distributed, as $(X, A)$, such that $\sup _{\mathbf{X} \in \mathcal{S}_{\mathbf{X}}}\left|\widehat{\pi}_{n}(\mathbf{X})-\pi_{\infty}(\mathbf{X})\right| \rightarrow 0$ a.s., for some function $\pi_{\infty}$ : $\mathbb{R}^{p} \rightarrow(0,1)$, where $\mathcal{S}_{\mathbf{X}}$ stands for the support of the distribution of $\mathrm{X}$.

- A2: $\inf _{\mathbf{X} \in \mathcal{S}_{\mathbf{X}}} \pi_{\infty}(\mathbf{X})=i_{\infty}>0$

- A3: There exists a sequence of random functions $\left(\widehat{g}_{n}\right)_{n \geq 1}, \widehat{g}_{n}: \mathbb{R}^{p} \rightarrow$ $\mathbb{R}$, depending on $\left(X_{1}, A_{1}, Y_{1}\right), \ldots,\left(X_{n}, A_{n}, Y_{n}\right)$, such that, for every compact set $\mathcal{K}, \sup _{\mathbf{X} \in \mathcal{K}}\left|\widehat{g}_{n}(\mathbf{X})-g_{\infty}(\mathbf{X})\right| \rightarrow 0$ a.s., for some function $g_{\infty}: \mathbb{R}^{p} \rightarrow \mathbb{R}$

Let $\phi=\mathbb{E}\left\{\pi(\mathbf{X}) / \pi_{\infty}(\mathbf{X})\right\}$, consider the distribution functions

$$
\begin{aligned}
& F_{1}(y)=\frac{1}{\phi} \mathbb{E}\left\{\frac{\pi(\mathbf{X})}{\pi_{\infty}(\mathbf{X})} \mathrm{I}_{\{Y \leq y\}}\right\}, \quad F_{2 a}(y)=\frac{1}{\phi} \mathbb{E}\left\{\frac{\pi(\mathbf{X})}{\pi_{\infty}(\mathbf{X})} \mathrm{I}_{\left\{g_{\infty}(\mathbf{X}) \leq y\right\}}\right\} \\
& F_{3 a}(y)=F_{g_{\infty}(\mathbf{X})}(y), \quad G(y)=F_{\left\{Y-g_{\infty}(\mathbf{X})\right\} \mid A=1}(y)
\end{aligned}
$$

and let

$$
F_{\infty}=F_{1}-F_{2 a} * G+F_{3 a} * G .
$$

The following result indicates in which circumstances $F_{\infty}$ coincides with $F_{0}$.

Theorem 1 Assume that the propensity score $\pi(\mathbf{X})=\mathbb{P}(A=1 \mid \mathbf{X})$ is equal to $\pi_{\infty}(\mathbf{X})$. Then, $F_{1}=F_{0}, F_{2 a}=F_{3 a}$ and therefore, $F_{\infty}=F_{0}$. Consider now the regression model $Y=g(\mathbf{X})+u$, with $u$ independent of $(A, \mathbf{X})$, and assume that $g(\mathbf{X})=g_{\infty}(\mathbf{X})$. Then, $F_{1}=F_{2 a} * G, F_{0}=F_{3 a} * G$ and consequently $F_{\infty}=F_{0}$. 
The next theorem establishes the double robustness of the quantile estimators.

Theorem 2 Assume that $Y=g(\mathbf{X})+u$, with $u$ independent of $(A, \mathbf{X})$, and let $\pi(\mathbf{X})$ denote the propensity score $\pi(\mathbf{X})=\mathbb{P}(A=1 \mid \mathbf{X})$. Let $\left\{\left(X_{i}, A_{i}, Y_{i}\right)\right\}_{i \geq 1}$ be independent and identically distributed as $(X, A, Y)$. Assume that conditions A1-A3 are satisfied, that the cumulative distribution function $G$ of $\left\{Y-g_{\infty}(\mathbf{X})\right\} \mid(A=1)$ is continuous. Assume also that either $g(\mathbf{X})=g_{\infty}(\mathbf{X})$ or $\pi(\mathbf{X})=\pi_{\infty}(\mathbf{X})$. Then

$$
\sup _{y}\left|\widehat{F}_{D P-N O R}(y)-F_{0}(y)\right| \rightarrow 0 \quad \text { a.s. }
$$

Moreover, $T_{p}\left(\widehat{F}_{D P-N O R}\right)$ is well defined and for every $p \in(0,1)$ such that $F_{0}$ is strictly increasing in a neighborhood of $T_{p}\left(F_{0}\right)$, we have

$$
T_{p}\left(\widehat{F}_{D P-N O R}\right) \rightarrow T_{p}\left(F_{0}\right) \text { a.s. }
$$

if $g(\mathbf{X})=g_{\infty}(\mathbf{X})$ or $\pi(\mathbf{X})=\pi_{\infty}(\mathbf{X})$.

\subsection{Robust Doubly Protected Estimators for Quan- tiles.}

We will now combine the robust notions discussed in Sections 3.4 with the consistency result presented in Section 4 to get a robust doubly protected estimator of the $p$-quantile $T_{p}\left(F_{0}\right)$. To do so, we consider parametric models $\pi(\mathbf{X} ; \boldsymbol{\gamma})$ and $g(\mathbf{X} ; \boldsymbol{\beta})$ for the propensity score and the regression function respectively and assume that at least one of them holds. Let $\widehat{\pi}_{n}(\mathbf{X})=\pi\left(\mathbf{X} ; \widehat{\gamma}_{n}\right)$, where $\widehat{\gamma}_{n}$ is the MLE under the postulated parametric model for the propensity score. The regression model will be fit with an MM- estimator, which will be denoted with $\widehat{\boldsymbol{\beta}}_{n}^{\mathrm{R}}$, and therefore, we define $\widehat{g}_{n}^{\mathrm{R}}(\mathbf{X})=g\left(\mathbf{X} ; \widehat{\boldsymbol{\beta}}_{n}^{\mathrm{R}}\right)$, while the residuals obtained from this procedure will be denoted with $\widehat{u}^{\mathrm{R}}$; that is to say, $\widehat{u}_{j}^{\mathrm{R}}=Y_{j}-g\left(\mathbf{X}_{j} ; \widehat{\boldsymbol{\beta}}_{n}^{\mathrm{R}}\right)$, for $j$ such that $A_{j}=1$. We propose to estimate the $p$ - quantile $T_{p}\left(F_{0}\right)$ with $T_{p}\left(\widehat{F}_{\mathrm{DP}-\mathrm{S} \text {-Rов }}\right)$, where $\widehat{F}_{\mathrm{DP}-\mathrm{S} \text { - вов }}$ is the following semiparametric normalized robust doubly protected estimator for $F_{0}$ :

$\widehat{F}_{\mathrm{DP}-\mathrm{S}-\mathrm{ROB}}=\frac{1}{C_{n}} \sum_{i=1}^{n} \frac{A_{i} \delta_{Y_{i}}}{\widehat{\pi}_{n}\left(\mathbf{X}_{i}\right)}-\frac{1}{C_{n} m} \sum_{i, j=1}^{n} \frac{A_{i}}{\widehat{\pi}_{n}\left(\mathbf{X}_{i}\right)} A_{j} \delta_{\widehat{g}_{n}^{\mathrm{R}}\left(\mathbf{X}_{i}\right)+\widehat{u}_{j}^{\mathrm{R}}}+\frac{1}{n m} \sum_{i, j=1}^{n} A_{j} \delta_{\widehat{g}_{n}^{\mathrm{R}}\left(\mathbf{X}_{i}\right)+\widehat{u}_{j}^{\mathrm{R}}}$,

The following theorem establishes that $T_{p}\left(\widehat{F}_{\text {DP-S-ROB }}\right)$ is a doubly protected estimator of $T_{p}\left(F_{0}\right)$. 
Theorem 3 Assume that $Y=g(\mathbf{X})+u$, with $u$ independent of $(A, \mathbf{X})$. Let $\left\{\left(X_{i}, A_{i}, Y_{i}\right)\right\}_{i \geq 1}$ be an i.i.d. sequence, distributed as $(X, A, Y)$. Denote with $\widehat{\boldsymbol{\gamma}}_{n}$ the MLE assuming a logistic regression model $\phi\left(\boldsymbol{\gamma}^{t} \mathbf{X}\right)$ for the propensity score $\mathbb{P}(A=1 \mid \mathbf{X})$. Let $\widehat{\boldsymbol{\beta}}_{n}^{R}$ be an MM-estimator, under the linear model $\boldsymbol{\beta}^{t} \mathbf{X}$ for the regression function $g(\mathbf{X})$. Assume that

(i) There exist $\boldsymbol{\gamma}_{\infty}$ and $\boldsymbol{\beta}_{\infty}^{R}$ such that $\widehat{\boldsymbol{\gamma}}_{n} \rightarrow \boldsymbol{\gamma}_{\infty}$ a.s. and $\widehat{\boldsymbol{\beta}}_{n}^{R} \rightarrow \boldsymbol{\beta}_{\infty}^{R}$ a.s.

(ii) $\mathcal{S}_{\mathbf{X}}$ is compact.

(iii) The cumulative distribution function $G$ of $\left.\left\{Y-\mathbf{X}^{t} \boldsymbol{\beta}_{\infty}^{R}\right)\right\} \mid(A=1)$ is continuous.

(iv) Either $\mathbb{P}(A=1 \mid \mathbf{X})=\phi\left(\boldsymbol{\gamma}_{0}^{t} \mathbf{X}\right)$, for some $\boldsymbol{\gamma}_{0}$, or $g(\mathbf{X})=\boldsymbol{\beta}_{0}^{t} \mathbf{X}$, for some $\boldsymbol{\beta}_{0}$.

Then

$$
T_{p}\left(\widehat{F}_{D P-S-R O B}\right) \rightarrow T_{p}\left(F_{0}\right) \text { a.s. }
$$

Remark 1 Both MLE and MM estimators are particular cases of $M$ - estimators and therefore, under regularity conditions, their limit point can be characterized, regardless the validity of the assumed model. In particular, under regularity conditions, the maximum likelihood estimator $\widehat{\gamma}_{n}$ converges a.s. to

$$
\boldsymbol{\gamma}_{\infty}=\arg \max _{\boldsymbol{\gamma}} \mathbb{E}\{\log p(\mathbf{X}, A, \boldsymbol{\gamma})\}
$$

where $p(\mathbf{X}, A, \gamma)=\pi(\mathbf{X} ; \boldsymbol{\gamma})^{A}\{1-\pi(\mathbf{X} ; \boldsymbol{\gamma})\}^{1-A}$, whether or not the postulated model for the propensity score is correctly specified. Similarly, whether or not the regression model is correctly specified, MM-estimators converge, under regularity conditions, to

$$
\boldsymbol{\beta}_{\infty}=\arg \min _{\boldsymbol{\beta}} \mathbb{E}\left(\rho\left[\{Y-\mathbf{X} \boldsymbol{\beta}\} / \sigma_{\infty}\right] \mid A=1\right),
$$

for some $\sigma_{\infty}>0$ (see Theorems 2 and 3 in 34 ).

\section{Monte Carlo Simulation}

In this section we report the results of a Monte Carlo study we made in order to analyze the performance of the different estimators of the median proposed in this work, as compared to some of the estimators that already exist in the literature. We consider different distributions of the error term $u$, namely standard normal and student distribution with one (Cauchy distribution) and 
three degrees of freedom. We also investigate the robustness of the proposed estimators, by contaminating the samples with $10 \%$ of outliers. We consider samples of $n$ i.i.d. random vectors $\left(\mathbf{X}_{i}, A_{i}, Y_{i}\right)$ where $\mathbf{X}_{i}=\left(1, X_{i 1}, X_{i 2}\right)$ is a bivariate standard normal random vector of covariates, that is, $\mathbf{X}_{i} \sim \mathcal{N}(\mathbf{0}, \mathbf{I})$, $A_{i}$ is a binary variable following a Bernoulli distribution with

$$
P(A=1 \mid \mathbf{X})=\pi(\mathbf{X})=\operatorname{expit}\left(\left(1, X_{1}, X_{2}\right) \gamma_{0}\right),
$$

where expit $(x)=e^{x} /\left(1+e^{x}\right), x \in \mathbb{R}$ and $\gamma_{0}^{t}=(0,0.1,-1.1)$; the outcome $Y$ satisfies

$$
Y=\left(1, X_{1}, X_{2}\right) \boldsymbol{\beta}_{0}+u,
$$

where $\boldsymbol{\beta}_{0}^{t}=(0,-3,2)$ and $u$ is independent of $(\mathbf{X}, A)$.

We consider four different situations:

S.1 Both the model for the propensity score and the model for the regression function are well specified.

S.2 The model for the propensity score is well specified while the model for the regression function is misspecified. More precisely, we fit an incorrect model for the regression function, using just the covariate $X_{1}$, that is, the covariate $X_{2}$ is omitted.

S.3 The model for the propensity score is misspecified while the model for the regression function is well specified. The misspecification consists in fitting a logistic regression model with only the covariate $X_{1}$, that is, the covariate $X_{2}$ is omitted.

S.4 Both models are misspecified by omitting the covariate $X_{2}$.

For each case, we generate $N r e p=1000$ samples of size $n=100$ and we compute 5 estimators of $\eta=\operatorname{med}(Y)$ by evaluating $T_{0.5}$ at different estimators of $F_{0}: \widehat{F}_{\text {IPW }}, \widehat{F}_{\mathrm{SY}}, \widehat{F}_{\mathrm{DP}-\mathrm{G}}, \widehat{F}_{\mathrm{DP}-\mathrm{G}-\mathrm{ROB}}$ and $\widehat{F}_{\mathrm{DP}-\mathrm{S}-\mathrm{ROB}}$. The definitions of these estimator can be found in equations (11), (23), (22), (24) and (36). Henceforth we will use the subscript of each of them to invoke the corresponding procedure; for instance IPw refers to the estimator $T_{0.5}\left(\widehat{F}_{\text {IPW }}\right)$. In each of the situations contemplated in S.1-S.4, $\widehat{\gamma}_{n}$ denotes the MLE computed under the postulated model for the propensity score; $\widehat{\boldsymbol{\beta}}_{n}$ and $\widehat{\boldsymbol{\beta}}_{n}^{\mathrm{R}}$ denote the least square estimator and an MM estimator for the proposed linear model for the regression function $g(\mathbf{X})$. As in [20], we take, as $\widehat{\boldsymbol{\beta}}_{n}^{\mathrm{R}}$, an MM-estimator, with $\rho_{0}$ and $\rho_{1}$ in the Tukey bisquare family, $k_{0}=1.57, k_{1}=3.44$ and $\delta=0.5$. In display (22) $\widehat{\sigma}$ stands for the clasical unbiased estimator of error standard deviation while $\widehat{s}$, in (24), is an MM scale estimator of the regression residuals. 
Empirical mean square errors are presented in Table 1, where PS stands for propensity score model, OR stands for outcome regression model, and the other three columns correspond to different distributions of the error term $u$ in the linear model. These results show that the double protection property of DP-G, DP-G-ROB and DP-S-ROB also holds for finite samples.

In order to investigate the robustness of the estimators, we contaminate with outliers samples generated as above, but with normal errors. To this end, $10 \%$ of the observations are replaced by outliers $\left(\mathbf{X}_{0}, A_{0}, Y_{0}\right)$ where $\mathbf{X}_{0}=$ $(1,2,0), \mathbb{P}\left(A_{0}=1 \mid \mathbf{X}=\mathbf{X}_{0}\right)=\operatorname{expit}\left((1,2,0) \boldsymbol{\gamma}_{0}\right)$ and

$$
Y_{0} \in\{-100,-90, \ldots,-20,-10,0,10,20, \ldots, 90,100\} .
$$

Simulation results under contaminations are summarized in Table 2 and Figures 1 to 3 . In Table 2 we show the maximum mean square error under $10 \%$ of contamination for values of $Y_{0}$ in the grid given in (37). Also in Table 2 , PS stands for propensity score model and OR for outcome regression model.

These results show that, even though the median is already robust, the estimation of the regression coefficients by a robust method improves the performance of the estimators. This improvement is very important if the sample is contaminated with outliers but it is also noticeable when the sample has a heavy tailed distribution such as a Student or a Cauchy distribution. On the other hand, DP-S-ROB gives better results than DP-G-ROB when the errors follow a Student or Cauchy distribution. This is due to the fact that the latter assumes normal errors while the former does not.

In figures 1 to 3 we plot the mean square errors of the different doubly protected estimators as a function of the value of the outlying outcome $Y_{0}$. These figures show that much robustness is gained by estimating the regression coefficients robustly, using an MM-estimator, instead of the least squares estimator. Both doubly protected robust estimators give good results for contaminated samples. Note that, as expected DP-G-ROB outperforms DP-s-Rов when the regression model is correctly specified, while DP-S-ROв outperforms DP-G-Rов when it is not.

\section{Example: Hospital data.}

We consider a sample of 100 patients hospitalized in a Swiss hospital during 1999 for medical back problems. We study the relationship between the cost of stay (Cost, in thousands of Swiss francs) and some explanatory variables that are available on administrative records: length of stay (LOS, in days), admission type $(0=$ planned; $1=$ emergency $)$, insurance type $(0=$ regular; 


\begin{tabular}{rrrrrr}
\hline Estimator & PS & OR & Normal errors & t3 errors & Cauchy errors \\
\hline IPW & correct & & 0.381 & 0.424 & 0.689 \\
IPW & incorrect & & 1.125 & 1.137 & 1.22 \\
SY & & correct & 0.206 & 0.233 & 0.339 \\
SY & & incorrect & 0.945 & 1.035 & 0.996 \\
DP-S-ROB & correct & correct & 0.313 & 0.361 & 0.548 \\
DP-S-ROB & correct & incorrect & 0.280 & 0.310 & 0.469 \\
DP-S-ROB & incorrect & correct & 0.683 & 0.528 & 0.841 \\
DP-S-ROB & incorrect & incorrect & 0.983 & 1.115 & 1.100 \\
DP-G & correct & correct & 0.310 & 0.361 & 0.707 \\
DP-G & correct & incorrect & 0.268 & 0.326 & 0.740 \\
DP-G & incorrect & correct & 0.712 & 0.570 & 0.733 \\
DP-G & incorrect & incorrect & 0.982 & 1.104 & 1.063 \\
DP-G-ROB & correct & correct & 0.310 & 0.364 & 0.590 \\
DP-G-ROB & correct & incorrect & 0.278 & 0.302 & 0.483 \\
DP-G-ROB & incorrect & correct & 0.682 & 0.537 & 0.942 \\
DP-G-ROB & incorrect & incorrect & 0.976 & 1.088 & 1.088 \\
\hline
\end{tabular}

Table 1: Mean square errors for different scenarios under the central model (without contaminations)

$1=$ private $)$, age (years $)$, sex $(0=$ female; $1=$ male $)$, discharge destination $(1=$ home; $0=$ another health institution $)$. This data set has been analyzed in [39] and has no missing values. In order to study the performance of our proposed estimators, we artificially delete some of the responses and compute the estimators in the sample with missing values. We repeat this procedure 1000 times

In each replication we generate a sample of dichotomous variables $A_{1} \ldots A_{n}$ according to the following mechanism:

$$
\ln \left(\frac{P\left(A_{i}=1\right)}{1-P\left(A_{i}=1\right.}\right)=0.1 * \mathbf{L O S}_{i}-1.1
$$

The responses with corresponding $A_{i}=0$ are deleted from the sample and considered missing. In this way, the proportion of missing responses is approximately 0.5 .

For each sample we compute estimators of the median Cost of stay by five methods: IPW, REG, DP-G, DP-G-ROB and DP-S-ROB. These estimates are compared with the median cost of stay computed with the entire sample, $\eta=9.69$, as follows: let $\widehat{\eta}$ be one of the five estimators mentioned above, then we estimate the mean square error of $\widehat{\eta}$ by 


\begin{tabular}{rrrr}
\hline Estimator & PS & OR & Max MSE \\
\hline IPW & correct & & 0.885 \\
IPW & incorrect & & 2.300 \\
SY & & correct & 1.641 \\
SY & & incorrect & 4.301 \\
DP-S-ROB & correct & correct & 0.675 \\
DP-S-ROB & correct & incorrect & 0.907 \\
DP-S-ROB & incorrect & correct & 0.733 \\
DP-S-ROB & incorrect & incorrect & 2.355 \\
DP-G & correct & correct & 1.036 \\
DP-G & correct & incorrect & 1.131 \\
DP-G & incorrect & correct & 2.706 \\
DP-G & incorrect & incorrect & 2.314 \\
DP-G-ROB & correct & correct & 0.695 \\
DP-G-ROB & correct & incorrect & 0.945 \\
DP-G-ROB & incorrect & correct & 0.681 \\
DP-G-ROB & incorrect & incorrect & 2.314 \\
\hline
\end{tabular}

Table 2: Maximum mean squared error under $10 \%$ of outlier contamination and the regression model with normal errors.

$$
M S E=\frac{1}{1000} \sum_{i=1}^{1000}\left(\widehat{\eta}_{i}-\eta\right)^{2},
$$

where $\widehat{\eta}_{i}$ is the value of $\widehat{\eta}$ at the $i-t h$ sample.

An analysis of the linear regression fit with the complete data set shows that all six variables considered are relevant to predict Cost and that no transformations are necessary; for this reason we consider this the "correct" model, both for the PS and the OR. To compare the fit with the one obtained if either model is misspecified, we also consider "incorrect" models, which include all six covariates, but LOS is transformed to logLOS.

The results are summarized in Table 3. This example suggests that both DP-S-ROB and DP-G-ROB have a good performance in real data sets with missing values, with better results than DP-G. DP-G-ROB is slightly better when the OR model is correctly specified, while DP-s-Rов is a somewhat more resistant to its misspecification.

\section{Acknowledgement}

The authors thank Dr. Alfio Marazzi for the data set in the example and Dr. Ana Bianco and Dr. Graciela Boente for helpful discussions. This 
research was partially supported by Grant PICT 2014-0351 from ANPCYT and Grants 20020150200110BA and 20020130100279BA from the Universidad de Buenos Aires at Buenos Aires, Argentina

\section{Appendix}

Proof of Theorem 1: If $\pi(\mathbf{X})=\pi_{\infty}(\mathbf{X})$, then $\pi(\mathbf{X}) / \pi_{\infty}(\mathbf{X})=1$ and $\phi=1$. Therefore, $F_{1}=F_{0}, F_{2 a}=F_{3 a}$ and $F_{\infty}=F_{0}$.

If $Y=g(\mathbf{X})+u$, with $u$ independent of $(A, \mathbf{X})$ and $g(\mathbf{X})=g_{\infty}(\mathbf{X})$, then $F_{3 a}$ is the distribution function of $g(\mathbf{X})$ and $G$ is the distribution function of $u$. Therefore, $F_{3 a} * G$ is the distribution function of $g(\mathbf{X})+u=Y$, that is to say $F_{3 a} * G=F_{0}$. On the other hand, let $Z$ be a random variable, independent of $u$, with distribution function $F_{2 a}$, then $F_{2 a} * G$ is the distribution function of $Z+u$, which, by definition, is equal to

$P(Z+u \leq y)=P(Z \leq y-u)=\frac{1}{\phi} \mathbb{E}\left\{\frac{\pi(\mathbf{X})}{\pi_{\infty}(\mathbf{X})} \mathrm{I}_{\{g(\mathbf{X}) \leq y-u\}}\right\}=\frac{1}{\phi} \mathbb{E}\left\{\frac{\pi(\mathbf{X})}{\pi_{\infty}(\mathbf{X})} \mathrm{I}_{\{g(\mathbf{X})+u \leq y\}}\right\}=F_{1}(y)$.

Thus, $F_{\infty}=F_{0}$ also in this case.

The following five lemmas will be used to prove Theorem 2, Recall that $\widetilde{F}_{1}$ and $\widetilde{F}_{2 a}$, defined in $(26)$ and $(27)$, respectively, are indeed random sequences of cumulative distribution functions based on sample of size $n$ (which we omit in the notation).

Lemma 1 Consider $\widetilde{F}_{1}$ and $F_{1}$, defined in (26) and (31), respectively. Under assumptions $A 1$ and $A 2$, it holds that $\widetilde{F}_{1}$ converges to $F_{1}$ uniformly, a.s., that is

$$
\mathbb{P}\left(\sup _{y}\left|\widetilde{F}_{1}(y)-F_{1}(y)\right| \rightarrow 0\right)=1
$$

Proof: We show first that $C_{n} / n \rightarrow \phi$ a.s. To do so, note that we can write

$$
\frac{C_{n}}{n}=\frac{1}{n} \sum_{i=1}^{n}\left\{\frac{A_{i}}{\widehat{\pi}_{n}\left(\mathbf{X}_{i}\right)}-\frac{A_{i}}{\pi_{\infty}\left(\mathbf{X}_{i}\right)}\right\}+\frac{1}{n} \sum_{i=1}^{n} \frac{A_{i}}{\pi_{\infty}\left(\mathbf{X}_{i}\right)}
$$

By the law of large numbers, the second term in (38) converges a.s. to

$$
\mathbb{E}\left\{\frac{A}{\pi_{\infty}(\mathbf{X})}\right\}=\mathbb{E}\left\{\frac{1}{\pi_{\infty}(\mathbf{X})} \mathbb{E}(A \mid X)\right\}=\mathbb{E}\left\{\frac{\pi(\mathbf{X})}{\pi_{\infty}(\mathbf{X})}\right\}=\phi
$$

It remains to prove that the first term in $(38)$ converges to zero a.s. Now, under conditions A1 and A2, given $\varepsilon \in(0,1)$ there exists $n_{0}$ such that 
$\left|\pi_{\infty}(\mathbf{X})-\widehat{\pi}_{n}(\mathbf{X})\right|<\varepsilon i_{\infty}$ for all $n \geq n_{0}$, and therefore, $(1-\varepsilon) i_{\infty} \leq \widehat{\pi}_{n}(\mathbf{X})$ for such $n$, implying that

$$
\frac{1}{n} \sum_{i=1}^{n} A_{i} \frac{\left|\pi_{\infty}\left(\mathbf{X}_{i}\right)-\widehat{\pi}_{n}\left(\mathbf{X}_{i}\right)\right|}{\widehat{\pi}_{n}\left(\mathbf{X}_{i}\right) \pi_{\infty}\left(\mathbf{X}_{i}\right)}<\frac{1}{n} \frac{1}{(1-\varepsilon) i_{\infty}^{2}} \sum_{i=1}^{n} A_{i}\left|\pi_{\infty}\left(\mathbf{X}_{i}\right)-\widehat{\pi}_{n}\left(\mathbf{X}_{i}\right)\right|<\frac{\varepsilon}{(1-\varepsilon) i_{\infty}}
$$

and then we obtain the announced result.

Second, we prove that

$$
\mathbb{P}\left\{\lim _{n \rightarrow \infty} \sup _{y}\left|\widetilde{F}_{1}(y)-\frac{1}{\phi} \frac{1}{n} \sum_{i=1}^{n} \frac{A_{i} \mathrm{I}_{\left\{Y_{i} \leq y\right\}}}{\pi_{\infty}\left(\mathbf{X}_{i}\right)}\right|=0\right\}=1 .
$$

To prove (40), notice that adding and subtracting $(n \phi)^{-1} \sum_{i=1}^{n} A_{i} \mathrm{I}_{\left\{Y_{i} \leq y\right\}} / \widehat{\pi}_{n}\left(\mathbf{X}_{i}\right)$, we get

$$
\left|\widetilde{F}_{1}(y)-\frac{1}{\phi} \frac{1}{n} \sum_{i=1}^{n} \frac{A_{i} \mathrm{I}_{\left\{Y_{i} \leq y\right\}}}{\pi_{\infty}\left(\mathbf{X}_{i}\right)}\right| \leq\left|\left\{C_{n} / n\right\}^{-1}-\phi^{-1}\right| C_{n} / n+\frac{1}{\phi n} \sum_{i=1}^{n} A_{i}\left|\widehat{\pi}\left(\mathbf{X}_{i}\right)^{-1}-\pi_{\infty}\left(\mathbf{X}_{i}\right)^{-1}\right| .
$$

Neither of the two terms in (41) depend on $y$ and they both converge to zero under A1-A2; the convergence of the first term follows from the convergence of $C_{n} / n$ to $\phi$ a.s., while the convergence of the second one has already been proved in (39). This proves (40).

Finally, using arguments similar to those in the proof of the GlivenkoCantelli theorem (see, for instance, Theorem 19.1 in [40] ), it can be shown that

$$
\mathbb{P}\left\{\lim _{n \rightarrow \infty} \sup _{y}\left|\frac{1}{n} \sum_{i=1}^{n} \frac{A_{i} \mathrm{I}_{\left\{Y_{i} \leq y\right\}}}{\pi_{\infty}\left(\mathbf{X}_{i}\right)}-\mathbb{E}\left\{\frac{A \mathrm{I}_{\{Y \leq y\}}}{\pi_{\infty}\left(\mathbf{X}_{i}\right)}\right\}\right|=0\right\}=1 .
$$

The result follows combining 40 and (42).

Henceforth we use $G_{n} \stackrel{w}{\rightarrow} G$ to denote weak convergence of cumulative distribution functions.

Lemma 2 Consider $\widetilde{F}_{2 a}$ and $F_{2 a}$, defined in (27) and (31), respectively. Under assumptions A1-A3, it holds that $\widetilde{F}_{2 a}$ converges weakly to $F_{2 a}$ a.s., i.e.,

$$
\mathbb{P}\left(\widetilde{F}_{2 a} \stackrel{w}{\rightarrow} F_{2 a}\right)=1
$$

Proof: Let $\mathcal{C}_{\text {buc }}$ denotes the set of functions $f: \mathbb{R} \rightarrow \mathbb{R}$ bounded and uniformly continuous. In order to prove the lemma we will show that 


$$
\mathbb{P}\left(\lim _{n \rightarrow \infty} \int f d \widetilde{F}_{2 a}=\int f d F_{2 a}, \forall f \in \mathcal{C}_{\text {buc }}\right)=1
$$

Let

$$
\widetilde{F}_{3}(y)=\frac{1}{C_{n}} \sum_{i=1}^{n} \frac{A_{i} \delta_{\widehat{g}_{n}\left(\mathbf{X}_{i}\right)}(y)}{\pi_{\infty}\left(\mathbf{X}_{i}\right)} \text { and } \widetilde{F}_{4}(y)=\frac{1}{C_{n}} \sum_{i=1}^{n} \frac{A_{i} \delta_{g_{\infty}\left(\mathbf{X}_{i}\right)}(y)}{\pi_{\infty}\left(\mathbf{X}_{i}\right)}
$$

Note that both $\widetilde{F}_{3}$ and $\widetilde{F}_{4}$ defined above, are sequences of random functions; however we omit $n$ in the notation for simplicity.

Fix $f \in \mathcal{C}_{\text {buc }}$. Defining $I_{1}(f)=\left|\int f d \widetilde{F}_{2 a}-\int f d \widetilde{F}_{3}\right|, I_{2}(f)=\left|\int f d \widetilde{F}_{3}-\int f d \widetilde{F}_{4}\right|$, and $I_{3}(f)=\left|\int f d \widetilde{F}_{4}-\int f d F_{2 a}\right|$, we get that

$$
\left|\int f d \widetilde{F}_{2 a}-\int f d F_{2 a}\right| \leq I_{1}(f)+I_{2}(f)+I_{3}(f) .
$$

Let us now consider each of these three terms. Since $f$ is bounded, using arguments similar to those in the proof of Lemma 1, we have that under A1 and A2

$$
\mathbb{P}\left(\lim _{n \rightarrow \infty}\left|\int f d \widetilde{F}_{2 a}-\int f d F_{3}\right|=0, \forall f \in \mathcal{C}_{\text {buc }}\right)=1 .
$$

To deal with $I_{2}(f)$ notice that

$I_{2}(f)=\left|\frac{1}{C_{n}} \sum_{i=1}^{n} \frac{A_{i} f\left\{\widehat{g}_{n}\left(\mathbf{X}_{i}\right)\right\}}{\pi_{\infty}\left(\mathbf{X}_{i}\right)}-\frac{1}{C_{n}} \sum_{i=1}^{n} \frac{A_{i} f\left\{g_{\infty}\left(\mathbf{X}_{i}\right)\right\}}{\pi_{\infty}\left(\mathbf{X}_{i}\right)}\right| \leq \frac{n}{C_{n}} \frac{1}{n i_{\infty}} \sum_{i=1}^{n}\left|f\left\{\widehat{g}_{n}\left(\mathbf{X}_{i}\right)\right\}-f\left\{g_{\infty}\left(\mathbf{X}_{i}\right)\right\}\right|($

Since $f$ is uniformly continuous, given $\varepsilon>0$, there exists $\delta$ such that $\mid u_{1}-$ $u_{2} \mid<\delta$ implies $\left|f\left(u_{1}\right)-f\left(u_{2}\right)\right|<\varepsilon$. Take $K$ large and consider the compact set $\mathcal{K}=\{\|\mathbf{X}\| \leq K\}$. For $n$ large enough, invoking now A3, we get that $\sup _{\mathbf{X} \in \mathcal{K}}\left|\widehat{g}_{n}(\mathbf{X})-g_{\infty}(\mathbf{X})\right|<\delta$ and therefore, the right hand side of 47) is smaller than

$$
\frac{n}{C_{n}}\left(\frac{\varepsilon}{i_{\infty}}+\frac{1}{n i_{\infty}} \sum_{i=1}^{n} 2\|f\|_{\infty} I_{\left\{\left\|\mathbf{X}_{i}\right\|>K\right\}}\right)
$$

which implies that

$$
\mathbb{P}\left(\lim _{n \rightarrow \infty}\left|\int f d \widetilde{F}_{3}-\int f d \widehat{F}_{4}\right|=0, \forall f \in \mathcal{C}_{\text {buc }}\right)=1 .
$$


It remains to show that

$$
\mathbb{P}\left(\lim _{n \rightarrow \infty} \int f d \widetilde{F}_{4}=\int f d F_{2 a}, \forall f \in \mathcal{C}_{\text {buc }}\right)=1
$$

Notice that, as in Lemma 1, using arguments similar to those in the proof of the Glivenko-Cantelli theorem, we have that

$$
\mathbb{P}\left(\lim _{n \rightarrow \infty} \sup _{y}\left|\frac{1}{n} \sum_{i=1}^{n} \frac{A_{i} \delta_{g_{\infty}\left(\mathbf{X}_{i}\right)}(y)}{\pi_{\infty}\left(\mathbf{X}_{i}\right)}-\mathbb{E}\left\{\frac{A \mathrm{I}_{\left\{g_{\infty}(\mathbf{X}) \leq y\right\}}}{\pi_{\infty}(\mathbf{X})}\right\}\right|=0\right)=1
$$

and therefore

$$
\mathbb{P}\left(\lim _{n \rightarrow \infty} \frac{1}{\widetilde{C}_{n}} \sum_{i=1}^{n} \frac{A_{i} \delta_{g_{\infty}\left(\mathbf{X}_{i}\right)}(y)}{\pi_{\infty}\left(\mathbf{X}_{i}\right)}=\frac{1}{\phi} \mathbb{E}\left\{\frac{A \mathrm{I}_{\left\{g_{\infty}(\mathbf{X}) \leq y\right\}}}{\pi_{\infty}(\mathbf{X})}\right\}, \forall y \in \mathbb{R}\right)=1(52)
$$

where $\widetilde{C}_{n}=\sum_{i=1}^{n} A_{i} / \pi_{\infty}\left(X_{i}\right)$. Both the sequence as the limit function presented in (52) are cumulative distribution functions. By the MAR assumption,

$$
\frac{1}{\phi} \mathbb{E}\left\{\frac{A \mathrm{I}_{\left\{g_{\infty}(\mathbf{X}) \leq y\right\}}}{\pi_{\infty}(\mathbf{X})}\right\}=F_{2 a}(y)
$$

and, therefore, (52) implies that

$$
\mathbb{P}\left(\lim _{n \rightarrow \infty} \frac{1}{\widetilde{C}_{n}} \sum_{i=1}^{n} \frac{A_{i} f\left(g_{\infty}\left(\mathbf{X}_{i}\right)\right)}{\pi_{\infty}\left(\mathbf{X}_{i}\right)}=\int f d F_{2 a}, \forall f \in \mathcal{C}_{\text {buc }}\right)=1
$$

Finally, since $\widetilde{C}_{n} / C_{n} \rightarrow 1$, we conclude that $(50)$ holds. The result stated in the lemma follows from combining (45), (46), (49) and (50).

The following lemma was proved in [20], as a part of Theorem 1.

Lemma 3 Consider $\widetilde{F}_{3 a}$ and $\widetilde{G}$, defined in $(29)$ and $(28), F_{3 a}$ and $G$ defined in (32). Under assumption $A 3, \widetilde{F}_{3 a}$ converges weakly to $F_{3 a}$ a.s. and also $\widetilde{G}$ converges wakly to $G$ a.s., i.e,

$$
\mathbb{P}\left(\widetilde{F}_{3 a} \stackrel{w}{\rightarrow} F_{3 a}\right)=1 \quad \text { and } \quad \mathbb{P}(\widetilde{G} \stackrel{w}{\rightarrow} G)=1 .
$$

As announced in Section 4, we will now show that the functional $T_{p}$, presented in (9), can be defined over an enlarged family of functions, which includes cumulative distribution functions, preserving its continuity. 
Lemma 4 Consider a distribution function $F: \mathbb{R} \rightarrow[0,1]$ and $p \in(0,1)$ such that there exists a unique value $y_{p}$ with $F\left(y_{p}\right)=p$, and so $T_{p}(F)=y_{p}$, for $T_{p}$ defined in (9). Let $F_{n}: \mathbb{R} \rightarrow \mathbb{R}, n \geq 1$, be a sequence of functions such that

1. $\lim _{y \rightarrow-\infty} F_{n}(y)=0$ and $\lim _{y \rightarrow+\infty} F_{n}(y)=1$.

2. $F_{n}$ converges uniformly to $F$.

Then $T_{p}$ can be defined at $F_{n}$ and

$$
\lim _{n \rightarrow \infty} T_{p}\left(F_{n}\right)=T_{p}(F)
$$

Proof: Let $A_{n, p}=\left\{y \in \mathbb{R}: F_{n}(y) \geq p\right\}$. By the assumptions of the lemma, $\lim _{y \rightarrow+\infty} F_{n}(y)=1$, and therefore, $A_{n, p}$ is not empty. Since $\lim _{y \rightarrow-\infty} F_{n}(y)=$ 0 we conclude that $A_{n, p}$ is bounded from below, and therefore $T_{p}\left(F_{n}\right)=$ $\inf A_{n, p}$ is well defined.

Given $\varepsilon>0$, let

$$
\left.\delta=\min \left\{\left(F\left(y_{p}+\varepsilon\right)-F\left(y_{p}\right)\right) / 2\right),\left(F\left(y_{0}\right)-F\left(y_{p}-\varepsilon\right)\right) / 2\right\} .
$$

By the assumptions of the lemma, $\delta>0$. Now, the uniform convergence of $F_{n}$ to $F$ guarantees that there exists $n_{0}$ such that

$$
\sup _{y \in \mathbb{R}}\left|F_{n}(y)-F(y)\right| \leq \delta, \text { for all } n \geq n_{0} .
$$

In particular,

$$
\sup _{y<y_{p}-\varepsilon} F_{n}(y)<F\left(y_{p}-\epsilon\right)+\delta \leq F\left(y_{p}\right)-2 \delta+\delta \leq p-\delta .
$$

and

$$
F_{n}\left(y_{p}+\varepsilon\right) \geq F\left(y_{p}+\varepsilon\right)-\delta \geq F\left(y_{p}\right)+2 \delta-\delta=p+\delta>p .
$$

From (55) and (56) we conclude that, for all $n \geq n_{0}$ we have $\left|y_{n}-y_{p}\right| \leq \delta$, and therefore, $y_{n} \rightarrow y_{p}$ en This concludes the proof.

Proof of Theorem 2: The continuity of $G$ implies that $F_{2 a} * G$ and $F_{3 a} * G$ are both continuous cumulative distribution functions. Since weak convergence to a continuous limit distribution function implies uniform convergence (see, for example, Lemma 2.11 in [40]), Lemmas 2 and 3 imply that 
$\widetilde{F}_{2 a} * \widetilde{G}$ and $\widetilde{F}_{3 a} * \widetilde{G}$ converge uniformly to $F_{2 a} * G$ and $F_{3 a} * G$, respectively, a.s.

Combining these results with Theorem 1, we obtain (34). From Lemma 4. we conclude that $T_{p}\left(\widehat{F}_{\mathrm{DP}-\mathrm{NOR}}\right)$ is well defined. Moreover, Lemma 4 and the uniform convergence proved bellow, implies that $T_{p}\left(\widehat{F}_{\text {DP-NOR }}\right)$ converges to $T_{p}\left(F_{0}\right)$ a.s.

\section{Proof of Theorem 3 :}

We will show that A1-A3 are satisfied, with $\widehat{\pi}_{n}(\mathbf{X})=\phi\left(\widehat{\gamma}_{n}^{t} \mathbf{X}\right), \pi_{\infty}(\mathbf{X})=$ $\phi\left(\boldsymbol{\gamma}_{\infty}^{t} \mathbf{X}\right), \widehat{g}_{n}(\mathbf{X})=\boldsymbol{\beta}_{n}^{t} \mathbf{X}$ and $g_{\infty}(\mathbf{X})=\boldsymbol{\beta}_{\infty}^{t} \mathbf{X}$. To prove A1, note that

$$
\left|\widehat{\pi}_{n}(\mathbf{X})-\pi_{\infty}(\mathbf{X})\right|=\left|\pi\left(\mathbf{X} ; \widehat{\boldsymbol{\gamma}}_{n}\right)-\pi\left(\mathbf{X} ; \boldsymbol{\gamma}_{\infty}\right)\right|=\left|\phi^{\prime}\left(\widetilde{\boldsymbol{\gamma}}_{n}^{t} \mathbf{X}\right) \mathbf{X}^{t}\left(\widehat{\boldsymbol{\gamma}}_{\boldsymbol{n}}-\boldsymbol{\gamma}_{\infty}\right)\right|
$$

where $\widetilde{\gamma}_{n}$ is an intermediate point between $\widehat{\gamma}_{n}$ and $\gamma_{\infty}$. The convergence of $\widehat{\gamma}_{n}$ to $\gamma_{\infty}$ a.s. combined with the assumed compactness for the support of $\mathbf{X}$ imply the validity of A1.

A2 is satisfied since $\phi\left(\gamma_{\infty}^{t} \mathbf{X}\right)$ is continuous and $\mathbf{X}$ has a compact support.

To prove the validity of $\mathrm{A} 3$, observe that

$$
\left|\widehat{g}_{n}(\mathbf{X})-g_{\infty}(\mathbf{X})\right|=\left|\left\{\widehat{\boldsymbol{\beta}}_{\boldsymbol{n}}-\boldsymbol{\beta}_{\infty}\right\}^{t} \mathbf{X}\right| \text {. }
$$

The convergence of $\widehat{\boldsymbol{\beta}}_{n}$ to $\boldsymbol{\beta}_{\infty}$ a.s. guarantees that A3 is also satisfied.

Finally, note that if $\mathbb{P}(A=1 \mid \mathbf{X})=\phi\left(\boldsymbol{\gamma}_{0}^{t} \mathbf{X}\right)$, then $\gamma_{\infty}=\gamma_{0}$, and so $\pi_{\infty}(\mathbf{X})=\mathbb{P}(A=1 \mid X)$. Also, if $g(\mathbf{X})=\boldsymbol{\beta}_{0}^{t} \mathbf{X}$, then $\boldsymbol{\beta}_{\infty}=\boldsymbol{\beta}_{0}$ implying that $g_{\infty}(\mathbf{X})=g(\mathbf{X})$. We can now invoke Theorem 2 to conclude the proof of the theorem.

\section{References}

[1] Little RJ, Rubin DB. Statistical Analysis with Missing Data. John Wiley and Sons 1987.

[2] Kang JD, Schafer JL. Demystifying double robustness: A comparison of alternative strategies for estimating a population mean from incomplete data. Statistical science 2007; 523-539.

[3] Little R, An H. Robust likelihood-based analysis of multivariate data with missing values. Statistica Sinica 2004; 949-968.

[4] Yates, F. The analysis of replicated experiments when the field results are incomplete. Emp. J. Exp. Agric. 1933; 1, 129-142. 
[5] Cheng, PE. Nonparametric estimation of mean functionals with data missing at random. Journal of the American Statistical Association 1994; $89,81-87$

[6] Imbens, G. W., Newey, W. K. and Ridder, G. . Mean-square-error calculations for average treatment effects. 2005

[7] Wang Q, Linton O and Hardle W. Semiparametric regression analysis with missing response at random. Journal of the American Statistical Association 2004; 99, 334-345.

[8] Robins JM, Rotnitzky A, Zhao LP. Analysis of semiparametric regression models for repeated outcomes in the presence of missing data. Journal of the American Statistical Association 1995; 90(429): 106-121.

[9] González-Manteiga, W. and Pérez-González, Nonparametric mean estimation with missing data. Comm. Statist. Theory Methods 2004; 33, 277-303.

[10] Lunceford JK, Davidian M. Stratification and weighting via the propensity score in estimation of causal treatment effects: a comparative study. Statistics in medicine 2004; 23 (19): 2937-2960.

[11] Carpenter J, Kenward, M and Vansteelandt, S. A comparison of multiple imputation and doubly robust estimation for analyses with missing data. Journal of the Royal Statistical Society. 2006; 169, 571-584.

[12] Bang H, Robins J. (2005). Doubly Robust Estimation in Missing Data and Causal Inference Models. Biometrics. 2005; 61, 962-972.

[13] Van der Laan, M. J. and Robins, J. M. Unified Methods for Censored Longitudinal Data and Causality. 2003; New York. Springer-Verlag.

[14] Cheng, P. E., Chu, C. K. Kernel estimation of distribution functions and quantiles with missing data. Statistica Sinica. 1996; 63-78.

[15] Yang, S., Kim, J. K., and Shin, D. W. Imputation methods for quantile estimation under missing at random. Statistics and Its Interface. 2013; $6(3), 369-377$.

[16] Qihua Wang and Yongsong Qin. Empirical likelihood confidence bands for distribution functions with missing responses. Journal of Statistical Planning and Inference. 2010; 140(9): 27782789. 
[17] Díaz, I.. Efficient estimation of quantiles in missing data models. Journal of Statistical Planning and Inference. 2017

[18] Bianco, , Boente, G., González-Manteiga, W., and Pérez-González, Estimation of the marginal location under a partially linear model with missing responses. Computational Statistics \& Data Analysis. 2010; 54(2), 546-564.

[19] Bianco, A, Boente, G, González-Manteiga, W and Perez Gonzalez, Ana. Asymptotic behaviour of robust estimators in partially linear models with missing responses:The effect of estimating the missing probability on the simplified marginal estimators. Test. 2011; 20 (3), 524-548.

[20] Sued M. and Yohai, V. Robust location estimation with missing data. Canadian Journal of Statistics. 2013; 41(1), 111-132.

[21] Zhang, Z., Chen, Z., Troendle, J. F. and Zhang, J. Causal inference on quantiles with an obstetric application. Biometrics. 2012; 68(3), 697-706.

[22] Rubin DB. Inference and missing data. Biometrika 1976; 63 (3): $581-592$.

[23] Rosenbaum PR, Rubin DB. The central role of the propensity score in observational studies for causal effects. Biometrika 1983; 41-55.

[24] Horvitz DG, Thompson DJ. A generalization of sampling without replacement from a finite universe. Journal of the American statistical Association 1952; 47(260): 663-685.

[25] Robins JM, Rotnitzky Recovery of information and adjustment for dependent censoring using surrogate markers. Aids Epidemiology. Springer 1992; 297-331

[26] Hirano K, Imbens GW, Ridder G. Efficient estimation of average treatment effects using the estimated propensity score. Econometrica 2003; 71(4): 1161-1189.

[27] Stone, C.J. Optimal rates of convergence for nonparametric estimators. Annals of Statistics 1980; 8, 1348-1360.

[28] Hastie T.J, Tibshirani RJ. Generalized Additive Models. Chapman and Hall 1990.

[29] Healy, M.J.R. and Westmacott,M. Missing values in experiments analyzed on automatic computers. Appl. Statist 1956. 
[30] Robins JM, Rotnitzky A, Zhao LP. Estimation of regression coefficients when some regressors are not always observed. Journal of the American statistical Association 1994; 89(427): 846-866.

[31] Scharfstein D, Rotnitzky a and Robins J. Adjusting for nonignorable drop-Out using semiparametric nonresponse Models. Journal of the American Statistical Association. 1999; 94, 448-499.

[32] Robins J and Rotnitzky Comment on the Bickel and Kwon article " Inference for semiparametric models: Some questions and an answer". Statistica Sinica. 2001; 11, 920-936.

[33] Yohai, V. J. High breakdown-point and high efficiency robust estimates for regression; The Annals of Statistics. 1987; 642-656.

[34] Fasano, M. V., Maronna, R. A., Sued, M., \& Yohai, V. J. Continuity and differentiability of regression M functionals. Bernoulli, 18(4), 2012; 1284-1309.

[35] Rosner B. Fundamentals of Biostatistics, 5th ed., Pacific Grove, CA: Duxbury 1999.

[36] Niu X, Hoff P. covreg: A simultaneous regression model for the mean and covariance. $\mathrm{R}$ package version 2014 .

[37] Neyman J. Sur les applications de la théorie des probabilités aux experiences agricoles: Essai des principes. Roczniki Nauk Rolniczych 1923; 10: $1-51$.

[38] Rubin DB. Estimating causal effects of treatments in randomized and nonrandomized studies. Journal of educational Psychology 1974; 66(5): 688.

[39] Marazzi, , Yohai, V. J. (2004). Adaptively truncated maximum likelihood regression with asymmetric errors. Journal of statistical planning and inference, 122(1), 271-291.

[40] Van der Vaart, W. (2000). Asymptotic statistics (Vol. 3). Cambridge university press.

[41] Maronna, R. A. R. D., Martin, R. D., \& Yohai, V. (2006). Robust statistics (pp. 978-0). John Wiley \& Sons, Chichester. ISBN. 


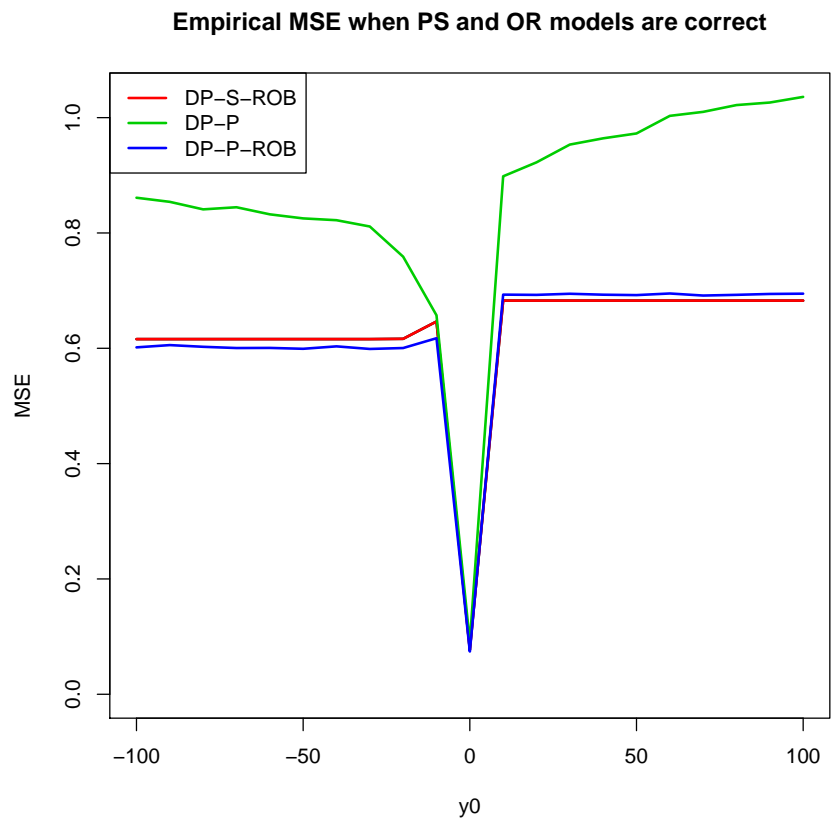

Figure 1: Doubly robust estimators in contaminated samples

\begin{tabular}{rrrr}
\hline Estimator & PS & OR & MSE \\
\hline IPW & correct & & 0.511 \\
IPW & incorrect & & 0.392 \\
SY & & correct & 0.107 \\
SY & & incorrect & 3.117 \\
DP-S-ROB & correct & correct & 0.146 \\
DP-S-ROB & correct & incorrect & 0.153 \\
DP-S-ROB & incorrect & correct & 0.127 \\
DP-S-ROB & incorrect & incorrect & 0.155 \\
DP-G & correct & correct & 0.225 \\
DP-G & correct & incorrect & 0.166 \\
DP-G & incorrect & correct & 0.288 \\
DP-G & incorrect & incorrect & 0.164 \\
DP-G-ROB & correct & correct & 0.142 \\
DP-G-ROB & correct & incorrect & 0.158 \\
DP-G-ROB & incorrect & correct & 0.122 \\
DP-G-ROB & incorrect & incorrect & 0.160 \\
\hline
\end{tabular}

Table 3: MSE of different estimators in different scenarios for Hospital data. 


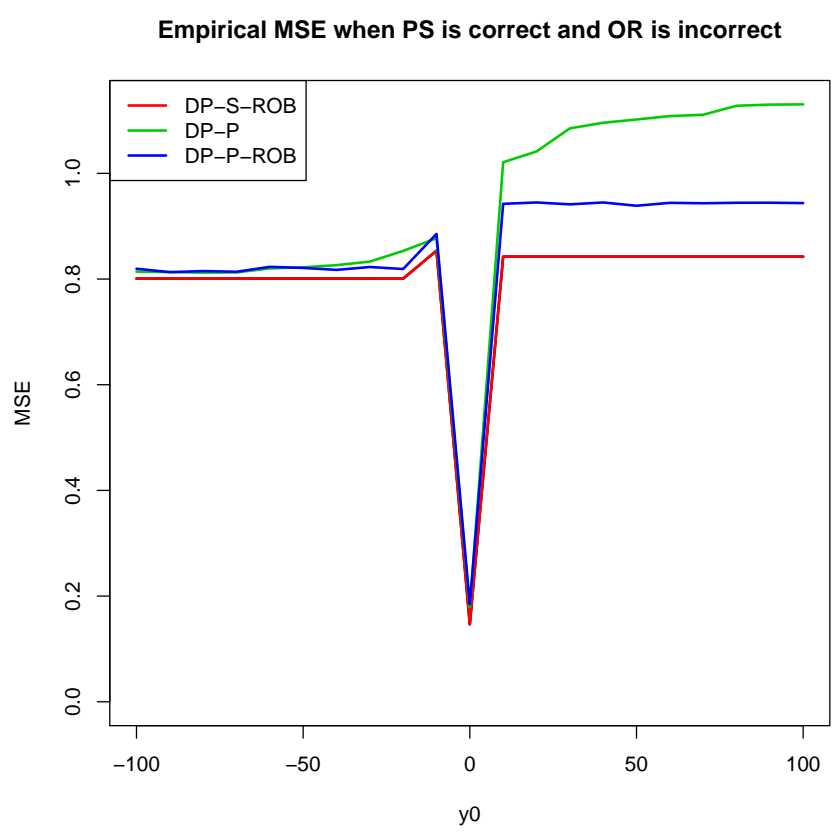

Figure 2: Doubly robust estimators in contaminated samples 


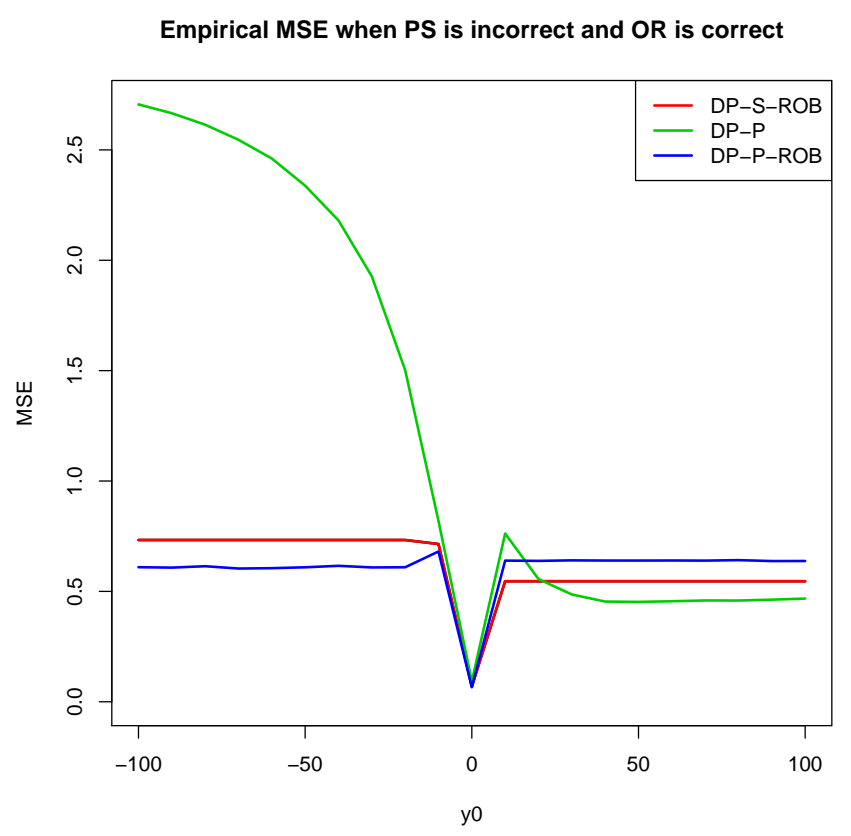

Figure 3: Doubly robust estimators in contaminated samples 